\title{
Investigating Sleep Fragmentation By Autonomic Arousals in Depressed Patients With Obstructive Sleep Apnea
}

\author{
Ahsan H Khandoker ${ }^{1}$, Taoufik Alsaadi ${ }^{2}$, Seada Kassie ${ }^{2}$, Leontios Hadjileontiadis ${ }^{1}$ \\ ${ }^{1}$ Khalifa University of Science and Technology, Abu Dhabi, UAE \\ ${ }^{2}$ American Center for Psychiatry and Neurology, Abu Dhabi, UAE
}

\begin{abstract}
The aim of this study is to look at if there is any difference of arousal patterns in polysomnography (PSG) and Pulse Transit Time (PTT) recordings in Obstructive Sleep Apnea (OSA) patients with Major Depressive Disorder (MDD) and without MDD subjects. Nine overnight PSG recordings (7 8 hours) were acquired from OSA subjects (AHI: 15 60; $36.68 \pm 13.55$ events/hour) with MDD diagnoses [OSAMDD](5 cases)

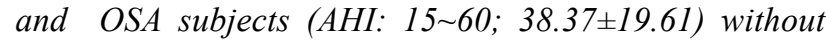
MDD [OSA] (4 cases) from a local psychiatric clinic. Selection criteria include the age [39.5 5 vs $41.4 \pm 3.5$ $y r s ; p>0.05]$ and weight $[120.2 \pm 10$ vs $118.6 \pm 12 \mathrm{Kgs}$; $p>0.05]$ matched subjects. Diagnoses of MDD were made by the Mini-International Neuropsychiatric Interview with ICD-10 questionnaire. A PTT arousal (ArPTT) was defined as a decrease in PTT by at least 15 milliseconds of the baseline lasting at least 5 seconds. Expert scored EEG based cortical arousals (ArEEG) include respiratory, movement, periodic leg movement and spontaneous arousals. Mann-Whitney U test showed that ArPTT index (events per hour) of OSA+MDD was significantly $(p<0.05)$ higher than that of OSA groups

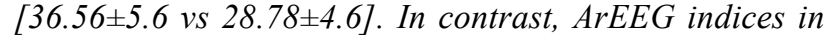
both groups were not found significantly different

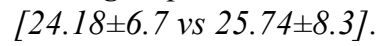

\section{Introduction}

Obstructive Sleep Apnea (OSA) is defined as the repetitive blockage of the upper airway, resulting in interrupted breathing during sleep. To restore breathing, arousal is needed to reopen the airway to activate the throat muscles. During sleep, the serotonergic system in the body regulates sleep through the release of serotonin and the activity of serotonergic neurons, which are at maximum during wakefulness, lower during slow wave sleep (SWS), and at minimum during REM sleep [1].

Major Depressive Disorder (MDD) is associated with a decrease in serotonergic and an increase in cholinergic neurotransmissions. So, antidepressants like selective serotonin reuptake inhibitors (SSRI) could activate the serotonergic system receptors causing an increase in the serotonergic activity and thus an inhibition of REM sleep. This could be one of the reasons why MDD patients often suffer from insomnia, early wake-ups and frequent awakenings during sleep. Compared to nondepressed subjects, depressed patients tend to have higher beta power, rapid eye movement (REM) activity, REM latency [2].

The relationship between Obstructive Sleep Apnea (OSA) and Major depressive disorder (MDD) is complex which may share neurological risk factors. Arousal (Ar) from sleep is an important mechanism for reestablishing upper airway patency in OSA. When EEG frequency changes are not seen at event termination by visible cortical arousals but accompanied by sudden drop in pulse transit time (PTT), those are known as autonomic arousals.

Pulse transit time (PTT) is a non-invasive cardiovascular measure which was used in the diagnosis of OSA in the past. PTT is the time taken for the arterial pulse pressure wave to travel from the left ventricle to a peripheral level (e.g. finger). The travel time of the pulse wave is inversely related to the arterial wall stiffness, which is determined by blood pressure. Hence, PTT may be inversely correlated to blood pressure [3]. The feasibility of PTT in detecting subcortical arousals and respiratory effort has been assessed in adults and recently also in children $[4,5,6]$. However, there are no studies on PTT based arousals in relation to mental illness such as MDD. Recently decreased arterial stiffness was correlated with acute and chronic mental stress [8], depression and anxiety [9]. Oulis and co-researchers documented reversal of arterial stiffness in a small group of severely depressed women, after a 6-week 
antidepressant therapy [Selective serotonin re-uptake inhibitors, Serotonin-norepinephrine reuptake inhibitors or a combination thereof in adequate dosages] [7], suggesting a pathophysiological connection.

It still remains to be answered if sleep quality measured by number of arousals per night sleep in OSA patients is different from OSA patients with co-morbidity of MDD. The aim of this study is to look at if there is any difference of arousal patterns in polysomnography (PSG) and PTT recordings in OSA patients with MDD and without MDD subjects.

\section{Methods}

\subsection{Subjects}

The overnight polysomnogram (PSG) data with psychiatric assessment profiles were collected from 9 patients at American Center for Psychiatry and Neurology (ACPN) in Abu Dhabi and analyzed by Miniscreen Software. The study protocol was approved by ACPN Ethics and Research Committee in 2017. Nine overnight PSG recordings (7 8 hours) were acquired from OSA subjects (AHI: 15 60; 36.68 \pm 13.55 events/hour) with MDD diagnoses [OSAMDD](5 cases) and OSA subjects (AHI: 15 60; 38.37 \pm 19.61$)$ without MDD [OSA] (4 cases) from a local psychiatric clinic. Selection criteria include the age [39.5 \pm 5 vs $41.4 \pm 3.5 \mathrm{yrs} ; \mathrm{p}>0.05]$ and weight [120.2 \pm 10 vs $118.6 \pm 12 \mathrm{Kgs}$; $\mathrm{p}>0.05]$ matched subjects. Diagnoses of MDD were made by the MiniInternational Neuropsychiatric Interview with ICD-10 questionnaire.

Each PSG study included electroencephalogram (channel C3-A2 and C4-A1), left and right electrooculogram (EOG), leg movements, body positions, thoracic and abdominal wall expansion (by respiratory inductive plethysmography), oronasal airflow (by nasal pressure), arterial oxygen saturation $\mathrm{SpO} 2$ (by pulse oximetry) and PPG (sampling frequency $=100 \mathrm{~Hz}$ with a resolution of 16 bits/sample). All subjects were free of any cardiac history. Diagnosis was based on clinical symptoms and PSG outcomes. Arousals were scored manually according to ASDA criteria using the EEG signal and marked using Miniscreen software.

\subsection{Pulse Transit Time (PTT)}

PTT was calculated as the time elapsing between the occurrence of the electrocardiographic R-wave and the point on the pulse waveform that is $50 \%$ of the maximum value [13].

\subsection{PTT based arousals}

PTT arousals (ArPTT) was defined as a fall in PTT of $\geq 15 \mathrm{~ms}$ lasting for $\geq 3 \mathrm{~s}[6,10,12]$. Ar PTT includes both respiratory and non-respiratory related arousals. Uninterpretable or spurious PTT signals extracted from artifacts in the electrocardiogram or pulse waveform were removed from the analysis.

Table 1: Patient Characteristics

\begin{tabular}{|l|l|l|}
\hline & OSA & OSA+MDD \\
\hline Number (N) & 5 & 4 \\
\hline Age (years) & $39.5 \pm 5$ & $41.4 \pm 3.5$ \\
\hline Weight (Kgs) & $120.2 \pm 10$ & $118.6 \pm 12$ \\
\hline AHI (events/hr) & $36.68 \pm 13.55$ & $38.37 \pm 19.61$ \\
\hline SWS of TST (\%) & $26.56 \pm 10.6$ & $27.6 \pm 12$ \\
\hline REM of TST (\%) & $20.4 \pm 6.7$ & $26.5 \pm 9.4^{*}$ \\
\hline
\end{tabular}

* significant difference at $\mathrm{p}<0.05$. SWS $=$ Slow Wave Sleep; $\mathrm{TST}=$ Total Sleep Time; REM= Retinal Eye Movement.

Table 2: Cortical (EEG based) ArEEG Microarousals (respiratory and non-respiratory related) [events/hour]

\begin{tabular}{|r|l|l|}
\hline & OSA & OSA+MDD \\
\hline Stage 1-2 & $5.4 \pm 3.2$ & $6.2 \pm 2.2$ \\
\hline SWS & $7.2 \pm 4.7$ & $4.3 \pm 7.5^{*}$ \\
\hline REM & $13.74 \pm 8.3$ & $15.18 \pm 6.7$ \\
\hline Total & $25.74 \pm 8.3$ & $24.18 \pm 6.7$ \\
\hline
\end{tabular}

Table 3: Autonomic (PTT based), ArPTT (Microarousals (respiratory and non-respiratory related) [events/hour]. $*=\mathrm{p}<0.05$.

\begin{tabular}{|r|l|l|}
\hline & OSA & OSA+MDD \\
\hline Stage 1-2 & $5.78 \pm 3.5$ & $4.34 \pm 2.1$ \\
\hline SWS & $7.78 \pm 5.4$ & $6.892 \pm 8.5$ \\
\hline REM & $18.78 \pm 4.6$ & $26.56 \pm 5.6^{*}$ \\
\hline Total & $28.78 \pm 4.6$ & $36.56 \pm 5.6^{*}$ \\
\hline
\end{tabular}

\section{Results and Discussion}

Figure 1 shows an example of arousal patterns of an OSA subject with MDD. Table 2 and 3 show that ArPTT index (events per hour) of OSA+MDD was significantly $(\mathrm{p}<0.05)$ higher than that of OSA groups [36.56 \pm 5.6 vs $28.78 \pm 4.6]$. In contrast, ArEEG indices in both groups were not found significantly different $[24.18 \pm 6.7$ vs $25.74 \pm 8.3]$.

In the previous research [15] it was reported that 
subcortical arousals due to increased upper airway resistance could be detected by PTT signals. Factually increased inspiratory resistive load is considered a main trigger mechanism for autonomic arousal and sleep disruption [13]. These upper airway resistance-related arousals apart from traditional respiratory events detected by PTT changes could be useful as a non-invasive diagnostic tool in the therapeutic management of MDD with OSA patients.

The relationship between autonomic arousals and the main factors affecting PTT is complex. The sympathetic nervous system is considered one of the major elements involved in the regulation of mean arterial pressure, affecting heart rate, left ventricular contractility, and systemic vascular resistance [14] and thereby PTT changes. How the magnitude of PTT is related to MDD is still unknown and remains to be investigated. It is however possible that the higher PTT based arousals could be related to changes in serotonergic pathways or other mechanical properties of the resistance of vessels of MDD patients.

However, understanding the neurobiological mechanism of autonomic arousals and serotonergic pathways needs further study.

\section{Acknowledgements}

This study was supported by Al Jalila Foundation grant awarded to Drs L. Hadjileontiadis and A.H. Khandoker. Fatima BaFakih, Noura Sayed, Fatima Alshamshi, Reem Alneyadi and Fatima Khulood Alameri created Fig 1 of the manuscript for their Capstone project.

\section{References}

[1] Zhang, X., Zheng, Y., Ren, Q., \& Zhou, H. (2017). The involvement of potassium channel ORK1 in shortterm memory and sleep in Drosophila. Medicine, 96(27).

[2] Adrien, J. (2002). Neurobiological bases for the relation between sleep and depression. Sleep Medicine Reviews, 6(5), 341-351.

[3] Smith RP, Argod J, Pepin JL, Levy PA (1999) Pulse transit time: an appraisal of potential clinical applications. Thorax 54:452- 457.

[4] Pitson DJ, Stradling JR (1998) Value of beat-to-beat blood pressure changes, detected by pulse transit time, in the management of the obstructive sleep apnoea/hypopnoea syndrome. Eur Respir J 12:685-692.

[5] Argod J, Pepin JL, Smith RP, Levy P (2000) Comparison of esophageal pressure with pulse transit time as a measure of respiratory effort for scoring obstructive nonapneic respiratory events. Am J Respir Crit Care Med 162:87-93
[6] Poyares D, Guilleminault C, Rosa A, Ohayon M, Koester U (2002) Arousal, EEG spectral power and pulse transit time in UARS and mild OSAS subjects. Clin Neurophysiol 113:1598-1606.

[7]Oulis, P., Kouzoupis, A., Kyrkou, K., Masdrakis, V. G., Georgiopoulos, G., Karapoulios, E., ... \& Papamichael, C. (2010). Reversal of increased arterial stiffness in severely depressed women after 6-week antidepressant treatment. Journal of Affective Disorders, 122(1), 164-166.

[8] Gorelick, P. B., Scuteri, A., Black, S. E., DeCarli, C., Greenberg, S. M., Iadecola, C., ... \& Petersen, R. C. (2011). Vascular contributions to cognitive impairment and dementia a statement for healthcare professionals from the American Heart Association/American Stroke Association. Stroke, 42(9), 2672-2713.

[9] Seldenrijk, A., van Hout, H. P., van Marwijk, H. W., de Groot, E., Gort, J., Rustemeijer, C., ... \& Penninx, B. W. (2011). Depression, anxiety, and arterial stiffness. Biological Psychiatry, 69(8), 795-803.

[10] Pitson D, Chhina N, Knijn S, van Herwaaden M, Stradling J (1994) Changes in pulse transit time and pulse rate as markers of arousal from sleep in normal subjects. Clin Sci (Lond) 87:269-273.

[11] Poyares D, Guilleminault C, Rosa A, Ohayon M, Koester U (2002) Arousal, EEG spectral power and pulse transit time in UARS and mild OSAS subjects. Clin Neurophysiol 113:1598-1606.

[12] Sleep Disorders Atlas Task Force of the American Sleep Disorders Association (1992) EEG arousals: scoring rules and examples. Sleep 15:173-184

[13] Pepin JL, Delavie N, Pin I, Deschaux C, Argod J, Bost $\mathrm{M}$ et al (2005) Pulse transit time improves detection of sleep respiratory events and microarousals in children. Chest 127:722-730, doi:10.1378/chest.127.3.722

[14] Charkoudian N, Rabbitts JA: Sympathetic Neural Mechanisms in Human Cardiovascular Health and Disease. Mayo Clinic Proceedings 2009, 84(9):822-830

[15] Katz ES, Lutz J, Black C, Marcus CL (2003) Pulse transit time as a measure of arousal and respiratory effort in children with sleep disordered breathing. Pediatr Res 53:580-588.

Address for correspondence. Name. Dr Ahsan Khandoker

Full postal address. Department of Biomedical Engineering, Khalifa University, Abu Dhabi, PO Box 127788, UAE.

E-mail address. ahsan.khandoker@ku.ac.ae 


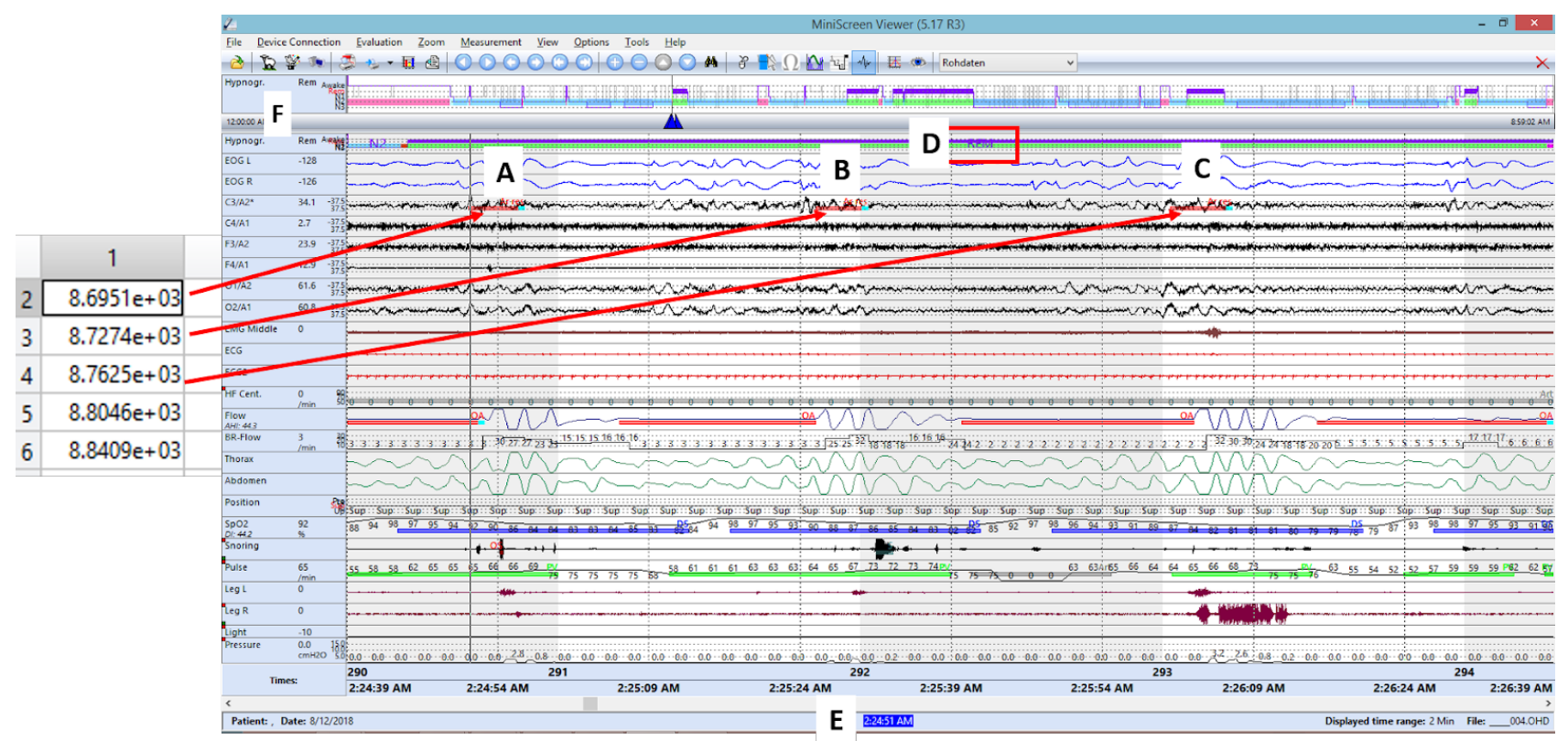

Figure 1: An screenshot of an OSA patient with MDD profile in Miniscreen software. A, B, and C indicates the timings of three arousals detected in the EEG signal during REM stage. $F$ indicates the hypnogram (sleep profile of the patient). 